\title{
Beauty and Astrophysics
}

\author{
Michael S. Bessell \\ Research School of Astronomy \& Astrophysics, Institute of Advanced Studies, \\ Australian National University, Private Bag, \\ Weston Creek PO, ACT 2611, Australia \\ bessell@mso.anu.edu.au,http://www.mso.anu.edu.au/ bessell
}

Received 1999 October 30, accepted 2000 April 10

\begin{abstract}
Spectacular colour images have been made by combining CCD images in three different passbands using Adobe Photoshop. These beautiful images highlight a variety of astrophysical phenomena and should be a valuable resource for science education and public awareness of science. The wide field images were obtained at the Siding Spring Observatory (SSO) by mounting a Hasselblad or Nikkor telephoto lens in front of a $2 \mathrm{~K} \times 2 \mathrm{~K} \mathrm{CCD}$. Options of more than 30 degrees or 6 degrees square coverage are produced in a single exposure in this way. Narrow band or broad band filters were placed between lens and CCD enabling deep, linear images in a variety of passbands to be obtained. We have mapped the LMC and SMC and are mapping the Galactic Plane for comparison with the Molonglo Radio Survey. Higher resolution images have also been made with the 40 inch telescope of galaxies and star forming regions in the Milky Way.
\end{abstract}

Keywords: techniques: image processing—surveys —miscellaneous

\section{Introduction}

\subsection{Foreword}

I chose this title of this paper to remind the jaded astronomers amongst us of the major reason why we chose astronomy as a career. It also explains why there are so many dedicated amateur astronomers and why the general public readily supports astronomy. Astronomy, combining beauty with mystery and wonder, remains a formidable combination of attractions for the minds of students and others with a restless intellect.

To a few theoreticians, $E=m c^{2}$ represents the highest form of beauty, but most of us are visual people stimulated more by pictures than equations. I well remember as a student attending Bart Bok's public lectures in Hobart and participating in his evocation of the wonders of astronomy through his black and white slides of the star-forming regions in Norma, Carina and the Magellanic Clouds. This was for me, I thought.

In the current world many of the best students are turning to economics and law and many students are more attracted to astrology and creationism than to mainstream science. In addition, within some of the liberal arts disciplines, there has been an increasing attack on the scientific method and the absolutism of science. It is therefore essential for the 'hard scientists', especially the physical scientists, to speak out in support of the scientific method of determining the truth in the physical world and to challenge the pessimistic outlook of the Jeremiahs in the community with the optimism from scientific discoveries both on Earth and elsewhere.

Part of this fight-back is not only to attract some of the best students back into mainstream science, but to educate all students and the general public about the importance of the scientific method and the importance of scientific research. A public better educated on these matters is more likely to result in governments that make well informed decisions and which will fund research and education adequately for the betterment of future generations.

School teachers, university lecturers and the media all have an important part to play in this and to assist them it is essential to provide a wide range of visual stimuli that can attract attention, maintain interest and affect the emotions. The coloured images from Mt Wilson, NASA, the Anglo-Australian Observatory (AAO) and more recently the Hubble space Telescope (HST) are excellent resources, but in this day of visual overload it is important to provide many new images of high impact that can be used for many different purposes and to provide these on a continuing basis.

\subsection{Stimulus and Opportunities}

In this paper I would like to give some of the results from imaging projects that I have been involved with at SSO using modest optics but with a large science grade CCD. I hope that this will encourage some of you to experiment with your own images and to utilise all these images that have been produced in your lectures and presentations.

The processing into colour images is the most time consuming and in many ways the most difficult step, albeit the most rewarding. The most exciting moment is the instant three different black and white images combine to produce colour. There are invariably surprises as, unlike earthly scenes of mountains, streams, skies and people that we see every day, we often have no idea of what colours or distribution of colours will appear in an astronomical image. I believe that maximum impact of an image requires the skills of a graphic designer/artist and an astronomer. There are few people born with these skills but it raises the possibility of collaborations. I can see many possible projects involving astronomers and creative artists and more specifically graphic design students, and even a major 
project for producing an interactive CDROM of the survey images.

Finally I hope that it will encourage amateur astronomers and teachers from schools with access to the new low and medium cost CCD systems to take on the challenging but very rewarding and creative astronomical colour imaging projects.

\subsection{The Many Uses of Colour}

There are many ways in which the use of colour enhances the information in an astronomical image. Although normal broad-band blue-green-red images approximating the eye's response can be used to illustrate many topics in astrophysics, it is the use of narrow-band images that provide more specific astrophysical information, particularly that involving the gas and dust in the interstellar medium. It is also the transformation of images made in passbands in the UV, IR or radio into optical colours that enhances our insights into astrophysical properties or highlights the unusual object. Such a technique has wide spread applications, one of which is to illustrate the photometric redshifts of distant galaxies. We also nowadays often make use of false-colour images to record intensity differences in an image made with a single passband. Colour is therefore the best way to present most data, but its use has been inhibited in the past by the expense of colour reproduction in the main science journals. However, with more and more electronic journals being produced, the provision of coloured JPG files should not be an added expense. David Malin (see e.g. Malin 1992) has pioneered the use of colour in astronomical imaging and you are refered to the AAO images page (www.aao.gov.au/images.html) for his suite of images derived from photographic plates and film, images that inspired the CCD work reported here.

\subsection{The Wide-field Imaging (WFI) Project}

I had been experimenting at the 16 inch telescope with the $2.3 \mathrm{~m}$ quartz optics reimaging camera and a $1 \mathrm{~K} \mathrm{CCD}$ to provide UBVI and $H \alpha$ images of the Magellanic Clouds. But because the scale was a little low and access to the imager camera was uncertain, John Hart suggested I try out a commercial Nikon telephoto lens bought many years earlier for an unsuccessful image tube project. The results were excellent. So, when Anne Green and Lawrence Cram made a request for $H \alpha$ images for comparison with their Molonglo Radio Surveys of the galactic plane (Green et al. 1999; see www.astrop.physics.usyd.edu.au/MGPS/) we decided that this was the appropriate setup. Funds were provided from Sydney University and the Institute for Advanced Studies at the Australian National University for a collaborative WFI project and a computer and data drive were purchased. A mechanical lens mount, filter holders and CCD mounting brackets were made and the Nikon imager providing a 6.8 degree field on a $2 \mathrm{~K} \mathrm{CCD}$ was installed on the 16 inch telescope mount in place of the existing telescope tube. Dome flat field screens and lamps were also provided. For the first year we used a CCD controller, computer and $2 \mathrm{~K} \mathrm{CCD}$ borrowed from the other SSO and Mt Stromlo Observatory (MSO) telescopes when they were unscheduled, but recently have obtained a dedicated $2 \mathrm{~K} \mathrm{CCD}$ and controller for the project. More details of the $H \alpha$ survey can be found at www.mso.anu.edu.au/ buxton/halpha.html.

We have also borrowed a Hasselblad lens from another ANU Department and made a new mounting so that we can image over 30 degrees of sky with a single exposure. We have also used the 40 inch telescope to obtain images at higher resolution of smaller objects, especially galaxies outside the local group.

Table 1 gives the mountings, cameras, image scales and field sizes.

Table 1. Camera focal lengths and fields

\begin{tabular}{cccccc}
\hline Name & Focal length & F no. & Aperture & Scale & Field \\
\hline Hasselblad & $80 \mathrm{~mm}$ & 2.8 & $60 \mathrm{~mm}$ & 60 arcsec & $30 \mathrm{deg}$. \\
Nikon & 400 & 4.5 & 90 & 12 & 6.8 \\
40 inch & 8128 & 8 & 1016 & 0.6 & 0.3 \\
\hline
\end{tabular}

\subsection{Acknowledgments}

Major contributors to the project have been Michelle Buxton (MSSSO) and Bob Watson (University of Tasmania). They did many of the initial observations and Bob developed all the IRAF scripts for the data processing. Other students from the Universities of Sydney, NSW and Wollongong have contributed to the observing, together with a team of amateurs from Coonabarabran organised by John Shobbrook who also wrote the observing manual. In 1999 Paul Price took over the $H \alpha$ survey as an honours project and Newcastle amateur Ken Hargreaves has done much observing. Ralph Sutherland (MSSSO) has been indispensable for his instructions on the use of Adobe Photoshop and his inspired image processing. The workshop staffs at SSO and MSO have been very supportive of the project both in its construction and operation. Hwankyung Sung provided many of the 40 inch images from his work on young star forming regions.

\section{Atlas of Images}

\subsection{Preamble}

Some examples of images from all these instruments will be shown. The images do not represent limiting observations but rather are illustrative of the information content and the astrophysical problems that they can address. The images are presented in a somewhat arbitrary progression from external galaxies to our galaxy, the Milky Way, then move along the galactic plane from Centaurus to Monocerotis commencing with the widest field views to the narrow views. The aim is to integrate what we see in our galaxy and the Magellanic Clouds with what we see in more distant galaxies. 
Table 2. List of Images

\begin{tabular}{|c|c|c|c|c|c|}
\hline No. & Name & Filters & Camera & Observer & Notes \\
\hline $1 \mathrm{a}$ & Antenna galaxies & $\mathrm{BVH \alpha}$ & 40 inch & $\mathrm{msb}$ & Pair of colliding galaxies. Note knots of star forming regions \\
\hline $1 b$ & Centaurus A & $\mathrm{BO} 3 H \alpha$ & 40 inch & b.w & Radio galaxy. Note green $[\mathrm{OIII}]$ jet top left \\
\hline $1 \mathrm{c}$ & M 95 & $\mathrm{BVH} H$ & 40 inch & $\mathrm{msb}$ & Theta galaxy. Note the low surface brightness outer arms \\
\hline $2 \mathrm{a}$ & NGC 1365 & $\mathrm{BVH} H$ & 40 inch & $\mathrm{msb}$ & Famous barred spiral. Note the dust lane leading into the core \\
\hline $2 b$ & NGC 2997 & $\mathrm{BVH} \alpha$ & 40 inch & $\mathrm{msb}$ & Grand Spiral. Note the HII regions in the spiral arms \\
\hline $2 \mathrm{c}$ & NGC 6744 & $\mathrm{BVH} \alpha$ & 40 inch & $\mathrm{msb}$ & A different type of barred spiral \\
\hline $3 \mathrm{a}$ & M 31 & $\mathrm{BVH} \alpha$ & Nikon & $\mathrm{msb}$ & Andromeda galaxy. Note the dust lanes and HII regions \\
\hline $3 b$ & Magellanic Clouds & $\mathrm{BVH} \alpha$ & Hasselb & $\mathrm{msb}$ & The closest galaxies to the Milky Way \\
\hline $3 \mathrm{c}$ & SMC & $\mathrm{BV} H \alpha$ & Nikon & $\mathrm{msb}$ & The SMC and wing + globular clusters 47 Tuc and NGC 362 \\
\hline $4 \mathrm{a}$ & SMC zoom & $\mathrm{BO} 3 H \alpha$ & Nikon & $\mathrm{msb}$ & The gas-rich SMC. The HII regions dominate in this image \\
\hline $4 b$ & LMC & $\mathrm{BO} 3 H \alpha$ & Nikon & $\mathrm{msb}$ & HII regions and the old stellar population in the bar \\
\hline $4 \mathrm{c}$ & LMC zoom & $\mathrm{BO} 3 H \alpha$ & Nikon & $\mathrm{msb}$ & 30 Doradus and shells of HII regions dominate this image \\
\hline $5 \mathrm{a}$ & 30 Doradus & BVR & 40 inch & sung & Three beautiful views of 30 Doradus. Stars dominate here \\
\hline $5 b$ & 30 Doradus & $\mathrm{BVH} \alpha$ & 40 inch & sung & In this image stars and the gas share the stage \\
\hline $5 c$ & 30 Doradus & $\mathrm{BO} 3 H \alpha$ & 40 inch & sung & Here the gas dominates. It appears 3 dimensional \\
\hline $6 a$ & Crux and pointers & $\mathrm{BV} H \alpha$ & Hasselb & $\mathrm{msb}$ & The archetypal view of the southern skies \\
\hline $6 b$ & Scorpio & $\mathrm{BVH} \alpha$ & Hasselb & $\mathrm{msb}$ & The huge constellation of Scorpio extends from the plane \\
\hline $6 c$ & Sag-Oph & $\mathrm{BV} H \alpha$ & Hasselb & $\mathrm{msb}$ & View looking toward the centre of the Milky Way \\
\hline $7 \mathrm{a}$ & Jewel Box & $\mathrm{BV} H \alpha$ & 40 inch & b.w & A young cluster of massive stars near the Southern Cross \\
\hline $7 \mathrm{~b}$ & NGC 2004 & $164 \mathrm{VH} \alpha$ & HST & b.dc.k & A similar cluster in the LMC with many times more stars \\
\hline $7 \mathrm{c}$ & NGC 2004 zoom & $164 \mathrm{VH} \alpha$ & HST & b.dc.k & These are HST images. Note the blue main sequence stars \\
\hline $8 \mathrm{a}$ & NGC 2100 zoom & $164 \mathrm{VH} \alpha$ & HST & b.dc.k & Be stars (pink), red supergiants (orange); \\
\hline $8 b$ & NGC 330 zoom & $164 \mathrm{VH} \alpha$ & HST & b.dc.k & A supergiant (white) \\
\hline $8 \mathrm{c}$ & NGC 346 & $\mathrm{BO} 3 H \alpha$ & 40 inch & $\mathrm{msb}$ & The largest cluster of young massive stars in the SMC \\
\hline $9 \mathrm{a}$ & Eta Carina & $\mathrm{BO} 3 H \alpha$ & Nikon & $\mathrm{msb}$ & The beautiful $\eta$ Car nebula. Note the lace work \\
\hline $9 b$ & Eta Carina & $\mathrm{BO} 3 H \alpha$ & 40 inch & $\mathrm{msb}$ & A closer view of the nebula and young clusters \\
\hline $9 \mathrm{c}$ & Eta Carina & $\mathrm{BVH} H$ & 40 inch & sung & Note the strange variable $\eta$ Car \\
\hline $10 \mathrm{a}$ & Trumpler 24 & $\mathrm{BV} H \alpha$ & 40 inch & sung & A young cluster and HII region \\
\hline $10 \mathrm{~b}$ & Bochum 14 & $\mathrm{BV} H \alpha$ & 40 inch & sung & An HII region surrounding a Wolf Rayet star \\
\hline $10 \mathrm{c}$ & Bochum 10 & $\mathrm{BVH} \alpha$ & 40 inch & sung & Another young cluster and HII region \\
\hline $11 \mathrm{a}$ & Lagoon \& Trifid & $\mathrm{BO} 3 H \alpha$ & Nikon & $\mathrm{msb}$ & The well known Lagoon and Trifid nebula \\
\hline $11 b$ & Trifid & $\mathrm{BO} 3 H \alpha$ & 40 inch & $\mathrm{msb}$ & A blue reflection nebula, HII region and dust lanes \\
\hline $11 \mathrm{c}$ & Lagoon zoom & $\mathrm{BO} 3 H \alpha$ & Nikon & $\mathrm{msb}$ & Colour change shows the ionisation gradient \\
\hline $12 \mathrm{a}$ & Lagoon inner & $\mathrm{BO} 3 H \alpha$ & 40 inch & $\mathrm{msb}$ & Yellow near the hottest stars. Red shows lower ionisation \\
\hline $12 \mathrm{~b}$ & Lagoon inner & $\mathrm{BV} H \alpha$ & 40 inch & sung & Beautiful dust lanes and starkly etched globules \\
\hline $12 \mathrm{a}$ & Lagoon inner zoom & $\mathrm{BO} 3 H \alpha$ & 40 inch & $\mathrm{msb}$ & Colours rotated to produce moody image-'a Turner' \\
\hline $13 \mathrm{a}$ & Eagle Nebula & $\mathrm{BO} 3 H \alpha$ & 40 inch & b.w & Star forming region HST image available \\
\hline $13 b$ & Eagle Nebula zoom & $\mathrm{BO} 3 H \alpha$ & 40 inch & b.w & Rotated field \\
\hline $13 \mathrm{c}$ & CG4 Nebula & BVR & 40 inch & $\mathrm{msb}$ & Interesting reflection nebula with dust clouds \\
\hline $14 \mathrm{a}$ & NGC 3603 & $\mathrm{BV} H \alpha$ & 40 inch & sung & Young cluster \\
\hline $14 \mathrm{~b}$ & NGC 6188 & $\mathrm{BO} 3 H \alpha$ & Nikon & b.w & Another interesting obscured nebulosity \\
\hline $14 \mathrm{c}$ & Pismis 24 & $\mathrm{BVH} H$ & 40 inch & sung & Young cluster \\
\hline $15 \mathrm{a}$ & IRAS & $10.60 .100 \mu$ & IRAS & IRAS & From Sutherland \& Bally \\
\hline $15 b$ & Orion-Eridanus & $\mathrm{BO} 3 \mathrm{H} \alpha$ & Hasselb & suth & Wide field view mosaic \\
\hline $15 \mathrm{c}$ & Orion & $\mathrm{BO} 3 H \alpha$ & Hasselb & $\mathrm{msb}$ & Showing Barnard's Loop around the Orion constellation \\
\hline $16 \mathrm{a}$ & $\lambda$ Orionis & $\mathrm{BO} 3 H \alpha$ & Nikon & $\mathrm{msb}$ & The 'bubble' surrounding the $O$ star $\lambda$ Orionis \\
\hline $16 b$ & Orion & $\mathrm{BO} 3 H \alpha$ & Nikon & $\mathrm{msb}$ & The region between the Belt and Sword of Orion \\
\hline $16 \mathrm{c}$ & Horsehead Nebula & $\mathrm{BO} 3 H \alpha$ & 40 inch & sung & The famous dark cloud in Orion \\
\hline $17 \mathrm{a}$ & Orion Nebula & $\mathrm{BO} 3 H \alpha$ & 40 inch & $\mathrm{msb}$ & Different views of the great nebula of Orion \\
\hline $17 \mathrm{~b}$ & Orion Nebula & $\mathrm{BVH} \alpha$ & 40 inch & $\mathrm{msb}$ & Different filters highlight small ionisation differences \\
\hline $18 \mathrm{a}$ & Orion Nebula & $\mathrm{S} 2 H \alpha \mathrm{N} 2$ & KPNO 1m & b.s & The seeing is much better in these next images \\
\hline $18 \mathrm{~b}$ & Orion Nebula & $\mathrm{S} 2 H \alpha \mathrm{N} 2$ & NTT $3.5 \mathrm{~m}$ & b.s & Note the detail in this excellent ground based image \\
\hline $18 \mathrm{c}$ & Orion Nebula & $\mathrm{S} 2 H \alpha \mathrm{N} 2$ & HST & b.s & Note the stellar tails all pointing away from the trapezium \\
\hline $19 \mathrm{a}$ & NGC $2264 \mathrm{~N}$ & $\mathrm{BVH} \alpha$ & 40 inch & sung & Part of young cluster in Monocerotis \\
\hline $19 b$ & NGC $2264 \mathrm{~W}$ & $\mathrm{BV} H \alpha$ & 40 inch & sung & Note the beautiful reflection nebula and dust clouds \\
\hline $19 \mathrm{c}$ & Rosette Nebula & $\mathrm{BO} 3 H \alpha$ & Nikon & murph & Strong ionisation produces these unusual colouring \\
\hline $20 \mathrm{a}$ & Vela-Puppis & $\mathrm{BO} 3 H \alpha$ & Hasselb & $\mathrm{msb}$ & This wide field view shows the comet Hale-Bopp lower right \\
\hline $20 \mathrm{~b}$ & Vela & $\mathrm{BO} 3 H \alpha$ & Nikon & $\mathrm{msb}$ & Note the $[\mathrm{OIII}]$ and $H \alpha$ filaments of the SN remnant \\
\hline $20 \mathrm{c}$ & Vela zoom & $\mathrm{BO} 3 H \alpha$ & Nikon & $\mathrm{msb}$ & Note the details of the twisted [OIII] filaments \\
\hline $21 \mathrm{a}$ & Crab Nebula & $\mathrm{BO} 3 H \alpha$ & 40 inch & b.w & Note the green [OIII], red $H \alpha$ and blue synchrotron light \\
\hline $21 b$ & Helix Nebula & $\mathrm{BO} 3 H \alpha$ & 40 inch & b.w & Planetary nebula. The blue and green colours have been reversed \\
\hline $21 \mathrm{c}$ & Comet Hale-Bopp & $\mathrm{V}$ & Nikon & $\mathrm{msb}$ & Note the two globular clusters. The colours represent intensities \\
\hline
\end{tabular}


The images of the external spiral galaxies show clearly the spatial distribution of the star forming regions, the giant HII regions (like red beads in a necklace) and the dust lanes lying along the arms and reaching right into the centre of the galaxies. The central bulges made up of older stars are also very obvious. When we look at the wide field images of the Milky Way we can recognise similar HII regions and dust lanes and we can then zoom in on these to reveal bright blue stars and shimmering curtains of ionised hydrogen. We can see the results of extensive stellar winds and the remnants of exploding stars with their delicate twisted shock fronts. By providing deep images of the interstellar region we can see the complicated interaction between hot stars and gas and marvel at the beauty of these interactions

The image scale (12 arcsec/pixel) with the Nikon lens is good for most large scale structure, but is unable to resolve the fine collimation of the Herbig-Haro jets associated with pre-main sequence stars or the disks of distant planetary nebulae, both of which are a couple of arcsec across. These are seen extremely well on the films of the new AAO Schmidt $H \alpha$ survey.

\subsection{Filters Used}

Many of the images combine two narrow band filters, $H \alpha$ (red) and [OIII] (green) with a short exposure B (blue). These bands are actually red (6563 $\AA$ ), green $(5007 \AA)$ and blue $(\approx 4300 \AA)$. The combination of HII $(13.6 \mathrm{eV})$ and [OIII] $(35.1 \mathrm{eV})$ with their significantly different ionisation potentials traces the ionisation gradients. It highlights the hottest stars and the most energetic shocks. A few images will be shown that feature more subtle ionisation differences using [SII], $H \alpha$ and [NII], all 'red' lines but shown as RGB in order of wavelength. Two images will also be shown with out-of-order colours that result in more striking images.

\subsection{List of Images}

The images are available for viewing from www.mso.anu.edu.au/ bessell/images/. Thumbnails of the images are displayed three per line for 21 lines. Table 2 lists the images and identifies them by line and position. It also gives the filters comprising the BGR of the colour image, the camera used (see Table 1), the observers, and a brief comment about the image or series of images. Detailed descriptions of many of the astronomical objects can be found with David Malin's images at www.aao.gov.au/images.html. The shorthand $\mathrm{O} 3, \mathrm{~S} 2, \mathrm{~N} 2$ is given for [OIII], [NII] and [SII] respectively. The code for the observers is msb: Bessell; b.w:
Buxton \& Watson; sung: Hwankyung Sung; murph: Murphy; b.dc.k: Bessell, Da Costa \& Keller; suth: Sutherland; and s.b: Sutherland \& Bally.

\section{Discussion}

\subsection{Are the Colours Real?}

The colours that are seen, are 'real' colours, in distinction to the 'false'colour images that one often sees these days. The blue, green and red colours are assigned to those actual observed colours in most cases; in the few cases where they are not they are at least assigned in the order of increasing wavelength. However, because the images utilise at least one narrow band filter centred on an emission line from ionised hydrogen, we 'see' the gas brighter than the star light by a factor that is the ratio of the width of the narrow band filter to the width of the broad band filter that represents the eye's colour band. That is, we increase the brightness of the hot interstellar gas in comparison to the brightness of the stars. We draw attention to the star forming regions by the effect that the hot stars have on the gas. We do not alter the relative colours of stars but we make them fainter in comparison to the brightness of the gas. But we do alter the colour of the gas depending on which emission line we choose to highlight.

For example, in the three images of 30 Doradus (line 5 ) we see very graphically the effect of changing the combination of filters used. Replacing the narrow green filter with a broad green filter weakens the green light from the gas compared to the starlight. Finally, replacing the narrow red filter with a broad red filter weakens the red light from the gas. The images shift from being star dominated to gas dominated and the colour of the gas changes while the colour of the stars stays the same.

In most of the images that are shown, by combining narrow band green $([\mathrm{OIII}])$ and narrow band red $(H \alpha)$ light, we produce a yellow colour when the [OIII] line is similar in strength to the $H \alpha$ line; we see this for example in the Lagoon and Trifid Nebulae (line 11) where the ionisation is produced by radiation from imbedded hot stars. Colour gradients between red and yellow show the varying strength of these lines as the radiation temperature of the gas changes.

However, in supernova remnants, like the Crab (line 21) and Vela (line 20) some of the ionisation results from high energy shocks and in these cases the [OIII] lines can be strong while $H \alpha$ is very weak. In that case the gas will appear green. We also see regions in SN remnants where there is a series of ionisation fronts, some green while others are yellow and red illustrating the complex ionisation structure and interaction between collisions and radiation. The use of [SII], $H \alpha$ and [NII] filters as seen in the series of Orion images from KPNO, NTT and HST (line 18) shows more subtle ionisation differences. 


\subsection{What is Reality in an Image?}

We distort 'reality' in many of our images in several ways. CCDs are linear detectors that faithfully record the relative brightnesses. However, our eyes are logarithmic detectors so is it more 'real' to process the CCD data to be logarithmic? We do this anyway in our data processing as explained below. But we need to do other distortions as well. The range of intensities that the eye can see, or the monitor or hard copy can provide, is limited. There is a great advantage in further compressing the intensity scale so as to keep detail in the bright areas of an image as well as in the fainter part. Photographic plates and prints did this a little by their non-linearity at the bright end but we can achieve it much better digitally. For example, in the images of galaxies (lines 1, 2 and 3) the nuclear regions and bulges of these galaxies tend to be much, much brighter than the outskirts. We have tried as hard as possible to retain the information in those bright regions, whilst enhancing the brightness and contrast in the outer spiral arm regions. The nuclear ring in NGC 2997 is just visible and the dust lanes with their imbedded star forming regions are seen in these barred spirals to reach into the central regions. Such details are not obvious in previously published images showing the outer regions.

Another way we distort reality, or rather we enhance reality, is by emphasising the hot interstellar regions. Normal broad-band optical images of galaxies and galactic fields mainly show stars. Within a narrow dynamic range these stars are coloured although the brighter ones are invariably white because the images are saturated. However, we know that in most galaxies, in particular in spiral galaxies and disk galaxies like the Milky Way and the Magellanic Clouds, the gas content is very high and in fact often dominates the mass distribution in the disk. Is it 'real' then to show a picture that mostly ignores a major physical component? By enhancing the faint light of ionised hydrogen as we have done in our images we are bringing to attention an important mass component. For example, in the wide field view of the Milky Way (line 6) we begin to see that the galactic plane is bathed in a sea of ionised gas and all the bright HII regions are probably connected. In the SMC and LMC (lines 3 and 4) we see galaxies that are dominated by gas, which is what they are. So these images bring a new perspective and in many ways are more 'real' than previous 'reality'. We still cannot see the neutral hydrogen but by combining the Parkes multibeam survey (www.atnf.csiro.au/research/multibeam/ multibeam.html) with these images, using another colour, we should be able to encompass that as well. So the difficulty and challenge is to present a beautiful image whilst retaining and emphasising the important physical information, the integrity of the object.

\subsection{Image Processing}

Finally, some specifics about the observing procedure and image processing. We aim to take three images through each filter with the telescope slightly offset between each exposure in the set of three. The three images through each filter are bias subtracted and flat fielded, then superimposed and combined by median filtering to produce a single grey image without cosmic rays and bad columns. The combined images through different filters are registered onto a reference image using the programs GEOMAP and GEOTRAN within IRAF. The 16 bit FITS files are then imported into PHOTOSHOP using a program written by Ralph Sutherland. This program, which generates 8 bit PHOTOSHOP data, samples the image and suggests upper and lower levels for the conversion. It also offers linear or logarithmic scaling. Most of the images shown here have had logarithmic scaling for all three colours (channels). (Sometimes special effects can be produced by combining logarithmic scaling in one or more colours with linear scaling in another.) We often need to reimport images with different levels chosen, especially near the sky to ensure that faint detail is not lost and to maximise the 256 levels in PHOTOSHOP by not wasting them.

In PHOTOSHOP the images are copied into the $\mathrm{R}, \mathrm{G}$ or B channel of a new image and then manipulated by adjusting levels, linearity and contrast. The manipulation of the levels and curves is where the artistic endeavour is required; however, one also needs to address what story is being told in order to determine how best to present the data. This is where the astronomy is needed together with the preconceived ideas of what a coloured astronomical picture should look like. Generally, we feel that the background sky should be dark with no dominant colour; that the bulk of the stars should be white or their colours soft shades of bluewhite, white, yellow or orange. Normally, blue stars come out blue-white, most stars come out white and red giants are generally deep yellow. The glowing interstellar region then produces its own unexpected colours. An image can of course be produced without any a priori astronomical knowledge and this would be an interesting exercise for an artist, but with one exception I will not present any such images here.

The HST images of the Magellanic cloud clusters (lines 7 and 8) show more subtle colours. Because the blue colour in the PHOTOSHOP image it represents the far ultraviolet colour of the star, the brightness of the blue image of the hot stars is much brighter than normal optical images. As a consequence, these stars come out very blue. But if those hot stars have strong $H \alpha$ emission (the Be stars), they appear pink in the colour image; you can see the high proportion of Be stars in these Cloud 
clusters. In addition, the great drop in UV light (compared to blue light) between an AF star and a KM star makes the AF stars white in the colour images and the KM stars orange.

We have made no attempt to produce real intensities in our manipulations. The intensity mappings are, however, monotonic although not linear. The manipulations are done for their impact without undermining the astrophysics. Where exact quantitative data are required this is best done with the individual linear monochromatic images in IRAF. We have generated good magnitudes or good stellar free $H \alpha$ images by subtracting or dividing the individual images in IRAF. Michael Murphy (UNSW) has also identified many probable planetary nebulae and Be stars in the Magellanic Clouds by doing photometry on $H \alpha$-[OIII] and [OIII]-continuum images (see Murphy \& Bessell 2000).

\subsection{Future Work}

Paul Price (Price 1999) is doing an honours project completing the $H \alpha$ survey and comparing it with specific portions of the the Molonglo Radio Survey
(Green et al. 1999) and the MSX IR Galactic Plane Survey (Cohen 1999) (http://gibbs1.plh.af.mil/). We are also completing the wide-field Hasselblad survey of the whole galactic plane visible from SSO and exploring with a commercial organisation the possibility of making an interactive CDROM using all the CCD images we have processed. However, generating colour images is very time consuming so it is unlikely that much more can be done without additional resources.

\section{Acknowledgments}

I would like to thank David Malin for suggestions that led to considerable improvements in the paper and for inspiring the pursuit of beauty in astronomical colour imaging.

\section{References}

Cohen, M. 1999, personal communication

Green, A. J., Cram, L. E., Large, M. I., \& Ye, T. 1999, ApJS, 122, 207

Malin, D. F. 1992, QJRAS, 33, 321

Murphy, M. E. \& Bessell, M. S. 1999, MNRAS, 311, 741

Price, P. A. 1999, personal communication 